\title{
Review Article \\ Statistical and Dynamical Decoupling of the IGM from Dark Matter
}

\author{
Li-Zhi Fang ${ }^{1}$ and Weishan Zhu ${ }^{1,2}$ \\ ${ }^{1}$ Department of Physics, University of Arizona, Tucson, AZ 85721, USA \\ ${ }^{2}$ Purple Mountain Observatory, Nanjing 210008, China \\ Correspondence should be addressed to Li-Zhi Fang, fanglz@physics.arizona.edu
}

Received 7 June 2010; Accepted 30 July 2010

Academic Editor: Martin Hardcastle

Copyright ( $) 2011$ L.-Z. Fang and W. Zhu. This is an open access article distributed under the Creative Commons Attribution License, which permits unrestricted use, distribution, and reproduction in any medium, provided the original work is properly cited.

Although the gravitational field in the universe is dominated by dark matter, cosmological observations show that the statistical properties of cosmic baryonic matter are significantly and systematically decoupled from that of the underlying dark matter. The dynamical reason of the decoupling is the difference of the nonlinear evolution of baryon fluid from that of collisionless dark matter. In highly nonlinear regime, the cosmic baryon fluid on scale free range evolves into the state of fully developed turbulence, of which the velocity field consists of shocks, vortices and complex structures. This scenario provides a coherent explanation of various phenomena referring to the statistical and dynamical decoupling of the IGM from dark matter.

\section{Introduction}

The mass field of the universe is dominated by dark matter, and only a tiny fraction of cosmic matter is in the form of baryonic particles. Gravitational clustering of dark matter is the major imputs of cosmic structure formation. Collapsed massive halos of dark matter are believed to be the hosts of various observed objects. On the other hand, light-emitting and absorbing objects are made up of baryonic matter.

Most of the baryonic matter in the universe actually is made of intergalactic gas or intergalactic medium (IGM), mainly consisting of neutral and ionized hydrogen and neutral and ionized helium [1-3]. For a long time, the mass field of the IGM, $\rho_{b}(\mathbf{r})$, is believed to be proportional to dark matter field $\rho_{\mathrm{dm}}(\mathbf{r})$. The similarity relation $\rho_{b}(\mathbf{r}) \propto \rho_{\mathrm{dm}}(\mathbf{r})$ should be hold, at least, on scales larger than the thermal diffusion or Jeans length of the IGM. In other words, the IGM mass field is considered as a point-by-point tracer of the dark matter distribution. Obviously, in this case, the baryon fraction $\rho_{b}(\mathbf{r}) /\left[\rho_{\mathrm{dm}}(\mathbf{r})+\rho_{b}(\mathbf{r})\right]$ is independent of $\mathbf{r}$, and equals to the cosmic mean $\Omega_{b} /\left(\Omega_{b}+\Omega_{\mathrm{dm}}\right)=0.17 \pm 0.01[4,5]$, where $\Omega_{b}$ and $\Omega_{\mathrm{dm}}$ are, respectively, the cosmological parameters of baryonic and dark matter.
However, the similarity $\rho_{b}(\mathbf{r}) \propto \rho_{\mathrm{dm}}(\mathbf{r})$ is found to be seriously inconsistent with observation in collapsed objects. The baryon fractions in gravitational bound objects generally are much lower than the cosmic mean. It raises the so-called missing baryon problem. The baryon fraction is claimed to decrease monotonically with the mass of collapsed halos. For $\mathrm{X}$-ray clusters and groups, the baryon fractions are smaller than the cosmic mean by a factor of $2-4$ [6-8]. The baryon fraction of dwarf galaxies can be as low as about $1 \%$ of the cosmic mean [9].

Many works have been carried out to explain the deficit of baryon content in gravitational collapsed objects, including feedback due to supernovae [10] or Active Galactic Nuclei (AGN) [11, 12], which blows out hot gas from halos, and preheating $[13,14]$, which heats baryon gas in the IGM and slows down the accretion of the IGM into halos. However, cosmologically hydrodynamic simulations show that these mechanisms fail to produce the baryon fraction in halos revealed by observation [15-17].

Actually, the baryon-dark matter decoupling probably is due mainly to the nonlinear nature of the hydrodynamics of the velocity and mass fields of IGM. That is, even without extra heating and cooling, the hydrodynamical evolution 
can already provide mechanisms of separating the baryonic matter from dark matter. A possible dynamical mechanism driving the baryonic matter deviate from dark matter has been addressed in the pioneer work of Shandarin and Zeldovich [18]. As the dark matter particles are assumed to be collisionless, their velocities would be multivalued at the intersection of trajectories. However, the baryon matter has a single-valued velocity field as a compressible fluid. Discontinuities, such as, shocks or complex structures, will develop inevitably in the baryon fluid at these intersections. Shocks would then cause the underdense of baryonic matter in the postshock area. On the other hand, dark matter is not affected by the shocks. Thus, discontinunities might prevent baryon matter to follow dark matter. Consequently, the baryon fraction in the collapsed halos would be less than cosmic mean [19].

Therefore, the nonlinear evolution of IGM would be a major reason of the statistical and dynamical decoupling between the cosmic baryon fluid and dark matter. We report the results of this mechanism. This paper will be arranged as follows. Section 2 is on the linear theory of evolution of IGM perturbations. Section 3 presents the decoupling in moderate nonlinear regime. Section 4 studies the fully nonlinear development of the IGM. It shows the cosmic baryon fluid to be turbulent on small scales. Section 5 gives observation evidences. The conclusions will be in Section 6 .

\section{Linear Evolution of the Perturbations of IGM Mass Field}

2.1. Basic Equations. Let us consider a flat universe having cosmic factor $a(t) \propto t^{2 / 3}$, and dominated by dark matter. We describe the IGM by a mass density field $\rho(\mathbf{x}, t)$ and a peculiar velocity field $\mathbf{v}(\mathbf{x}, t)$, where $\mathbf{x}$ is the comoving coordinate. In hydrodynamical description, the equations of the IGM consist of the continuity and the momentum (or the Eular) equations as [20]

$$
\begin{gathered}
\frac{\partial \delta}{\partial t}+\frac{1}{a} \nabla \cdot(1+\delta) \mathbf{v}=0, \\
\frac{\partial a \mathbf{v}}{\partial t}+(\mathbf{v} \cdot \nabla) \mathbf{v}=-\frac{1}{\rho} \nabla p-\nabla,
\end{gathered}
$$

where the density perturbation of the IGM $\delta(\mathbf{x}, t)=$ $[\rho(\mathbf{x}, t)-\bar{\rho}] / \bar{\rho}$, and $\bar{\rho}$ is the mean density of the IGM. $p$ is the pressure of the IGM.

The IGM can be considered as a "passive substance" with respect to the underlying dark matter. Namely, the evolution of the IGM mass field $\rho(\mathbf{x}, t)$ is governed by the gravity of total matter (baryonic plus dark matter). The peculiar gravitational potential $\phi$ in (2) satisfies

$$
\nabla^{2} \phi=4 \pi G a^{2} \bar{\rho}_{t} \delta_{t}
$$

where the operator $\nabla$ is acting on the comoving coordinate $\mathbf{x}$, and $\delta_{t}(\mathbf{x}, t)=\left[\rho_{t}(\mathbf{x}, t)-\bar{\rho}_{t}\right] / \bar{\rho}_{t}$ is the perturbation of total density field, with mean density $\bar{\rho}_{t}=1 / 6 \pi G t^{2} \propto a^{-3}$. The potential $\phi$ will be zero (or constant) when density perturbation $\delta_{t}=0$.
To sketch the gravitational clustering of the IGM, we will not consider the details of heating and cooling. Thermal processes generally are local, and therefore, it is reasonable to describe the thermal processes by a polytropic relation $p(\mathbf{x}, t) \propto \rho^{\gamma}(\mathbf{x}, t)$. Thus (2) can be rewritten as

$$
\frac{\partial a \mathbf{v}}{\partial t}+(\mathbf{v} \cdot \nabla) \mathbf{v}=-\frac{\gamma k_{B} T}{\mu m_{p}} \frac{\nabla \delta}{(1+\delta)}-\nabla \phi,
$$

where the parameter $\mu$ is the mean molecular weight of the IGM particles, and $\mathrm{m}_{p}$ is the proton mass. In this case, we do not need energy equation, and the IGM temperature evolves as $T \propto \rho^{\gamma-1}$ or $T=T_{0}(1+\delta)^{\gamma-1}$. Exactly speaking, there is also a noise in the hydrodynamical (2) and (4), which are related to the viscous of the IGM fluid due to the fluctuationdissipation theorem. Since the IGM is a "passive substance", this noise would be ignorable in searching for the deviation of the IGM from the underlying dark matter.

2.2. Linear Solution of Growth Mode. The unperturbed solutions of the density and velocity fields of baryonic matter are $\bar{\rho}=\left[\Omega_{b} /\left(\Omega_{b}+\Omega_{\mathrm{dm}}\right)\right] \bar{\rho}_{t} \propto a^{-3}$ and $\mathbf{v}=0$. Therefore, the linearization of (1) and (4) yields

$$
\begin{gathered}
\frac{\partial \delta}{\partial t}+\frac{1}{a} \nabla \cdot \mathbf{v}=0, \\
\frac{\partial a \mathbf{v}}{\partial t}=-\frac{\gamma k_{B} \bar{T}}{\mu m_{p}} \nabla \delta-\nabla,
\end{gathered}
$$

where the mean temperature $\bar{T} \propto \bar{\rho}^{\gamma-1} \propto a^{-3(\gamma-1)}$. In the Fourier space, we have

$$
\begin{gathered}
\frac{\partial^{2} \delta(\mathbf{k}, t)}{\partial t^{2}}+2 \frac{\dot{a}}{a} \frac{\partial \delta(\mathbf{k}, t)}{\partial t}+\frac{1}{t^{2}} \frac{k^{2}}{k_{J}^{2}} \delta(\mathbf{k}, t)=4 \pi G \bar{\rho} \delta_{t}(\mathbf{k}, t), \\
\frac{\partial v(\mathbf{k}, t)}{\partial t}+\frac{\dot{a}}{a} v(\mathbf{k}, t)=-\frac{1}{t^{2} a} \frac{1}{k_{J}^{2}} \delta(\mathbf{k}, t)+\frac{4 \pi G \bar{\rho} a}{k^{2}} \delta_{t}(\mathbf{k}, t)
\end{gathered}
$$

where $\mathbf{v}(\mathbf{k}, t)=i \mathbf{k} v(\mathbf{k}, t)$, and the Jeans wavenumber $k_{j}$ is given by $k_{J}^{2}=\left(a^{2} / t^{2}\right)\left(\mu m_{p} / \gamma k_{b} \bar{T}\right) \propto a^{3 \gamma-4}$. When $\gamma=4 / 3, k_{J}$ is time-independent. Here, we ignore the stochastic nature of (6), and the randomness of all the variables is given by the random initial conditions.

We consider only the growth mode of the perturbation of dark matter, that is, $\delta(k, t) \propto a$. In the case of $\gamma=4 / 3$, the solution of (6) is [21]

$$
\delta(\mathbf{k}, t)=\frac{\delta_{t}(\mathbf{k}, t)}{1+3 k^{2} / 2 k_{J}^{2}}+c_{1} t^{-(1+\epsilon) / 6}+c_{2} t^{-(1-\epsilon) / 6}
$$

where $\epsilon=\left(1-4 k^{2} / 9 k_{J}^{2}\right)^{1 / 2}$, and constants $c_{1}$ and $c_{2}$ depend on the initial condition $\delta(\mathbf{k}, 0)$ and $v(\mathbf{k}, 0)$. Therefore, regardless of the initial condition of the IGM, after a long evolution we always have

$$
\delta(\mathbf{k}, t)=\delta_{t}(\mathbf{k}, t) \quad \text { if } k \ll k_{J} .
$$


This means that the evolution of the IGM will completely follow dark matter, and the initial difference between $\delta(\mathbf{k}, t)$ and $\delta_{t}(\mathbf{k}, t)$ will be forgotten. Equation (8) yields

$$
P_{b}(k)=P_{\mathrm{dm}}(k) \quad \text { if } k \ll k_{J} .
$$

The power spectrum of IGM field, $P_{b}(k)$, has to be the same as that of dark matter $P_{\mathrm{dm}}(k)$.

The linear solutions of the IGM have also been studied by using different assumptions of the IGM thermal processes other than that used in $(4)[22,23]$. A common feature of these solutions is

$$
\begin{aligned}
\delta(\mathbf{k}, t)= & (1+\text { decaying terms }) \delta_{t}(\mathbf{k}, t) \\
& + \text { decaying terms } \text { if } k \ll k_{J} .
\end{aligned}
$$

Only the decaying terms are affected by the initial conditions, and therefore, the solutions of equations (8) and (9) hold in general, regardless of the specific assumptions of the IGM thermal processes.

The physical explanation of the solutions (8) and (10) is straightforward. The IGM is only a tiny component in the cosmic mass field and its evolution is completely governed by the gravity of dark matter in the linear regime. After the decaying of the initial conditions, the IGM should follow the same trajectory as dark matter on scales larger than the Jeans length.

In linear approximation, we also have the linear relation as follows:

$$
\delta(\mathbf{x}, t)=-f \nabla \cdot \mathbf{v}(\mathbf{x}, t),
$$

where the parameter $f$ depends on dark matter models [24].

\section{Moderate Nonlinear Regime of the IGM Evolution}

3.1. Burgers' Equation for Cosmic Baryonic Fluid. In the moderate regime, we may use linear relation (11) for $\delta$ in (4). We have then

$$
\frac{\partial a \mathbf{v}}{\partial t}+(\mathbf{v} \cdot \nabla) \mathbf{v}=-\frac{\nu}{a} \nabla^{2} \mathbf{v}-\nabla \phi,
$$

where the coefficient $v$ is given by

$$
v=\frac{\gamma k_{B} T_{0}}{\mu m_{p}(d \ln D(t) / d t)}
$$

in which $D(t)$ describes the linear growth behavior. The term with $v$ in (12) describes a diffusion which is characterized by the Jeans length $k_{J}$.

To sketch the evolution of gravitational clustering, only the growth modes are of interest. For the growth modes, the velocity field is irrotational, and we can define a velocity potential by

$$
\mathbf{v}=-\frac{1}{a} \nabla \varphi
$$

Substituting (14) into (12), we have

$$
\frac{\partial \varphi}{\partial t}-\frac{1}{2 a^{2}}(\nabla \varphi)^{2}-\frac{\nu}{a^{2}} \nabla^{2} \varphi=\phi .
$$

Equation (15) is the stochastic-force-driven Burgers' equations or the KPZ equations [25]. It is the simplest nonlinear Langevin equation for growth modes [26-28]. This equation can also be found with a coarse-graining approximation [29].

Thus, in moderate nonlinear regime, the cosmic baryonic fluid is similar to Burgers' fluid. The second term on the 1.h.s. results from convection, and the third on the l.h.s. describes relaxation of the clustering by diffusion. The term on the r.h.s represents the gravitational potential of dark matter, which provides the initially random perturbations. Clustered structures will develop from the initial seeds via the competition of the convection and diffusion.

3.2. IGM as a Burgers' Fluid. The Burgers' equation contains two scales: the dissipation length or the Jeans length $1 / k_{J}$ and the correlation length $r_{c}$ of the random force, $\phi$. The intensity of the random force $\phi$ can be characterized by the density contrast of dark matter $\delta_{\mathrm{dm}}$. Many theoretical works on Burgers fluids have been done [30-34]. They show that turbulence will develop in Burger fluid when the following Reynolds number is large [33, 35]:

$$
\left(k_{J} r_{c}\right)^{2 / 3}\left\langle\delta_{\mathrm{dm}}^{2}\right\rangle^{1 / 3}>1 \text {. }
$$

This condition corresponds to the system in which the dark matter field has entered the nonlinear regime. In the linear regime, $\delta_{\mathrm{dm}} \ll 1$, and therefore, perturbations on scale $r_{c}>1 / k_{J}$ will not cause Burgers' turbulence in the IGM. In the moderate nonlinear regime, $\delta_{\mathrm{dm}} \geq 1$, and Burgers' turbulence on scales larger the Jeans length can develop. Since the dark matter field becomes nonlinear first on small scales and then on large scales, one can expect that Burgers' turbulence in the IGM develops from small to large scales.

When Burgers turbulence develops in the IGM, its velocity and mass density fields will be intermittent and contain discontinuities or shocks. The probability distribution function (PDF) of velocity field is long tailed [33]. Since dark matter is not affected by Burgers turbulence, the IGM velocity field will dynamically decouple from the dark matter field on scales larger than the Jeans length once Burgers turbulence is developed. The intermittence of the IGM mass field, as a result of the Burgers equation, has also been emphasized in [23].

Jeans diffusion will also lead to the decoupling between the velocity fields of the IGM $\mathbf{v}$ and dark matter $\mathbf{v}_{\mathrm{dm}}$. However, the decoupling given by the Jeans diffusion is very different from that results from the Burgers turbulence shocks. For the former, the distribution $\mathbf{v}$ and $\Delta \mathbf{v} \equiv \mathbf{v}(\mathbf{x}+$ $\mathbf{r})-\mathbf{v}(\mathbf{x}-\mathbf{r})$ is symmetric with respect to the transformation $\mathbf{v}_{\mathrm{dm}} \rightarrow-\mathbf{v}_{\mathrm{dm}}$, that is, the velocity PDF with $\Delta \mathbf{v} \cdot \mathbf{v}_{\mathrm{dm}} /\left|\mathbf{v}_{\mathrm{dm}}\right|>$ 0 (acceleration in the direction of $\mathbf{v}_{\mathrm{dm}} /\left|\mathbf{v}_{\mathrm{dm}}\right|$ ) is the same as the PDF with $\Delta \mathbf{v} \cdot \mathbf{v}_{\mathrm{dm}} /\left|\mathbf{v}_{\mathrm{dm}}\right|<0$ (deceleration in the direction of $\mathbf{v}_{\mathrm{dm}} /\left|\mathbf{v}_{\mathrm{dm}}\right|$ ). For Burgers turbulence, the shocks consist of an acceleration ramp followed by a rapid deceleration. Therefore, the IGM is not symmetric between the 
sections accelerating and decelerating. Since the acceleration is due to the gravity of dark matter, the acceleration is generally in the direction of $\mathbf{v}_{\mathrm{dm}}$. Thus, the IGM velocity field $\mathbf{v}$ is asymmetric with respect to the transformation $\mathbf{v}_{\mathrm{dm}} \rightarrow$ $-\mathbf{v}_{\mathrm{dm}}$. The PDF of $\Delta \mathbf{v}$ with $\Delta \mathbf{v} \cdot \mathbf{v}_{\mathrm{dm}} /\left|\mathbf{v}_{\mathrm{dm}}\right|>0$ will not be the same as the PDF of $\Delta \mathbf{v}$ with $\Delta \mathbf{v} \cdot \mathbf{v}_{\mathrm{dm}} /\left|\mathbf{v}_{\mathrm{dm}}\right|<0$.

If $v=0$, Burgers equation (15) is scale-free when the random force $\phi$ is scale-free. In this case, the velocity field of the Burgers fluid is self-similar and the PDF of the velocity difference $\Delta \mathbf{v}$ is scale-invariant.

In summary, if the IGM can be described as a Burgers' fluid, its velocity field should show the following properties: (1) the peculiar velocity of the IGM at a given point will generally be lower than that of dark matter at the same point, (2) the PDF of $\Delta \mathbf{v}_{r} \cdot \mathbf{v}_{\mathrm{dm}} /\left|\mathbf{v}_{\mathrm{dm}}\right|>0$ will not be the same as the PDF of $\Delta \mathbf{v}_{r} \cdot \mathbf{v}_{\mathrm{dm}} /\left|\mathbf{v}_{\mathrm{dm}}\right|<0$, and (3) the PDF of $\Delta \mathbf{v}_{r}$ is scaling. These predictions have been proven by cosmologically hydrodynamic simulation samples [36].

\section{Fully Developed Nonlinear Regime}

4.1. Vorticity. Burgers turbulence is qualitatively different from turbulence described by the Navier-Stokes equations. The latter generally consists of vortices on various scales, while the former is a collection of shocks. These features arise because growth modes of the perturbations of velocity fields are potential, and the velocity field is irrotational. Moreover, from the condition (16), we see that the Burgers turbulence can only develop in dense areas of dark matter $\delta_{\mathrm{dm}}>1$.

However, in fully developed nonlinear regime, the cosmic baryon fluid will on longer be potential, and the turbulence of Navier-Stokes fluid can also be developed in high as well as low density regions. This point can be seen with the vorticity, which is defined by $\omega_{i}=(1 / 2)\left(\partial_{i} v_{j}-\partial_{j} v_{i}\right)$ or $\vec{\omega}=\nabla \times \mathbf{v}$, where $i=1,2,3$. The dynamical equation of the vorticity $\vec{\omega}$ of cosmic baryon fluid can be derived from Navier-Stokes equations as follows [37]:

$$
\begin{aligned}
\frac{D \vec{\omega}}{D t} & \equiv \partial_{t} \vec{\omega}+\frac{1}{a} \mathbf{v} \cdot \nabla \vec{\omega} \\
& =\frac{1}{a}\left(\mathbf{S} \cdot \vec{\omega}-d \vec{\omega}+\frac{1}{\rho^{2}} \nabla \rho \times \nabla p-\dot{a} \vec{\omega}\right),
\end{aligned}
$$

where $d=\partial_{i} \mathrm{v}_{i}$ is the divergence of the velocity field. Tensor $\mathbf{S}$ is called strain rate defined as $S_{i j}=(1 / 2)\left(\partial_{i} v_{j}+\partial_{j} v_{i}\right)$, and the vector $[\mathbf{S} \cdot \vec{\omega}]_{i}=S_{i j} \omega_{j}$.

A remarkable property of (17) is the absence of the term of gravity of mass fields, indicating that the gravitational field of dark matter is not the sources of the vorticity. This point is obvious, as gravity is curl-free in nature. In this sense, the vorticity is "nongravitational".

In the linear regime of perturbations, both the terms S $\cdot \vec{\omega}-d \vec{\omega}$ and $\nabla \rho \times \nabla p$ would be zero. Vorticity $\vec{\omega}$ will decay as $a^{-1}$, given by the term $\dot{a} \vec{\omega}$. Therefore, as expected, in the linear regime the velocity field of baryon matter is irrotational. In other words, the linear evolution of the velocity field of baryon fluid is fully governed by the gravity.

However, in the fully developed nonlinear regime, the vorticity will no longer be zero. First, it has been shown that at the moderate nonlinear regime, Burgers' fluid will inevitably lead to the development of shocks and complex structures, which can yield nonzero term $\nabla \rho \times \nabla p$ of (17), being called baroclinity. Thus, the vorticity is excited, regardless of the gravity of dark matter. Physically, once multistreaming has developed, complex structures, like curved shocks, will lead to deviation of the direction of $\nabla p$ from that of $\nabla \rho$. Consequently, the density pressure relation cannot be simply given by a single-variable function equation as $p=p(\rho)[38]$ and the baroclinity term will no longer be zero.

Once vorticity is initiated, (17) shows that the vorticity of baryon velocity field can be maintained by itself, that is, the system actually is nonlinearly self-excited and selfmaintained. The divergence $d=\partial_{j} v_{j}$ is generally negative in regions of clustering. Therefore, the term $-d \omega$ of (17) will lead to an amplification of vorticity in overdense regions. On the other hand, the vorticity will be stretched by the nonlinear term $S \cdot \vec{\omega}$. Therefore, vorticity can also develop in low mass density region. This picture is very different from the Burgers' turbulence.

Vortices are considered as a fundamental ingredient of turbulence. The fluctuations of the vorticity field is an important measurement of the turbulence of fluid [39, 40]. Therefore, the dynamics of vorticity provides a nongravitational mechanism of the decoupling between baryon matter from dark matter.

The turbulence of cosmic baryon fluid can also be seen with the spectrum tensors $\Phi_{i j}(\mathbf{k})$ and $\Omega_{i j}(\mathbf{k})$, which are, respectively, the Fourier counterparts of the two-point correlation tensors of velocity $\left\langle v_{i}(\mathbf{x}+\mathbf{r}) v_{j}(\mathbf{x})\right\rangle$ and vorticity $\left\langle\omega_{i}(\mathbf{x}+\mathbf{r}) \omega_{j}(\mathbf{x})\right\rangle$. That is,

$$
\begin{gathered}
\Phi_{i j}(\mathbf{k})=\frac{1}{(2 \pi)^{3}} \int\left\langle v_{i}(\mathbf{x}+\mathbf{r}) v_{j}(\mathbf{x})\right\rangle e^{-i \mathbf{k} \cdot \mathbf{r}} d \mathbf{r}, \\
\Omega_{i j}(\mathbf{k})=\frac{1}{(2 \pi)^{3}} \int\left\langle\omega_{i}(\mathbf{x}+\mathbf{r}) \omega_{j}(\mathbf{x})\right\rangle e^{-i \mathbf{k} \cdot \mathbf{r}} d \mathbf{r},
\end{gathered}
$$

respectively, where $\langle\cdots\rangle$ denotes average over spatial coordinates $\mathbf{x}$. For a homogeneous turbulence, we have [39]

$$
\Omega_{i j}(\mathbf{k})=\left[\delta_{i j} k^{2}-k_{i} k_{j}\right] \Phi_{l l}(\mathbf{k})-k^{2} \Phi_{i j}(\mathbf{k}),
$$

and hence,

$$
\Omega_{i i}(\mathbf{k})=k^{2} \Phi_{i i}(\mathbf{k})
$$

The power spectra of velocity and vorticity fields are defined, respectively, as

$$
\begin{aligned}
& P_{v}(k)=\int \frac{1}{2} \Phi_{i i}(\mathbf{k}) \delta(|\mathbf{k}|-k) d \mathbf{k}, \\
& P_{\omega}(k)=\int \frac{1}{2} \Omega_{i i}(\mathbf{k}) \delta(|\mathbf{k}|-k) d \mathbf{k} .
\end{aligned}
$$

Combining (20) and (21) yields

$$
P_{\omega}(k)=k^{2} P_{v}(k)
$$


This relation can be used to measure the developed level of turbulence. If the velocity and vorticity fields of a fluid satisfy the relation given by (22), it should be in the state of fully developed homogeneous turbulence. Otherwise, it would be less developed. The samples of cosmological hydrodynamic simulation have showed that the relation (22) is perfectly hold on scales less than $3 \mathrm{~h}^{-1} \mathrm{Mpc}$ at $z \sim 0$ [37]. Actually, the dynamics of (17) reveals that the cosmic baryonic fluid underwent a scale-free evolution, which leads to a state of fully developed turbulence.

4.2. She-Leveque Intermittence. The velocity field of a fully developed turbulence is highly intermittent, which is measured by the structure functions $S_{p}(r)$ defined as

$$
S_{p}(r) \equiv\left\langle\delta v_{r}^{p}\right\rangle \sim r^{\zeta_{p}}
$$

where $\delta v_{r} \equiv[(\mathbf{v}(\mathbf{x}+\mathbf{r})-\mathbf{v}(\mathbf{x})) \cdot \mathbf{r} / r]$ and $\zeta_{p}$ is called intermittent exponent. Based on dimensional argument of hierarchical evolution, Kolmogorov in 1941 [41] predicted that for fully developed turbulence, the intermittent exponent is $\zeta_{p}=p / 3$ on scales of inertial range. Experimental and numerical results do not, however, support the $p / 3$ law. It should be attributed to intermittency, that is, turbulence field in inertial range is characterized by stronger non-Gaussianity on smaller scales. A remarkable development was made by She and Leveque (SL hereafter) [42]. They proposed that the non-Gaussian behavior of fully developed turbulence is determined by the hierarchical structure originated from the Navier-Stokes equation, and the $p / 3$ law should be replaced by

$$
\zeta_{p} / \zeta_{3}=\left[1-C\left(1-\beta^{3}\right)\right] \frac{p}{3}+C\left(1-\beta^{p}\right)
$$

which contains only two dimensionless parameters $C$ and $\beta$. $C$ is the Hausdorff dimension of the most dissipative structures, and parameter $\beta$ is given by the hierarchical evolution. SL formula (24) is in excellent agreement with various experiments of turbulence, including turbulence in compressible fluid. The SL scaling law of structure function is considered to be universal for characterizing the fully developed turbulence.

With the hydrodynamic simulation sample of the concordance $\Lambda \mathrm{CDM}$ universe, the intermittent exponent of the velocity field of cosmic baryon fluid at redshift $z=0$ in the scale range from the Jeans length to about $16 \mathrm{~h}^{-1} \mathrm{Mpc}$ is found to be extremely well described by the She-Leveque's universal scaling formula (24) with $C=1$ and $\beta^{3}=1 / 3$ [43]. It indicates that the dissipative structures are dominated by sheets. These results imply once again that the evolution of highly evolved cosmic baryon fluid is similar to a fully developed turbulence.

When a fluid is turbulent, the kinetic energy passes from large to the smallest eddies, and finally dissipates into thermal motion. For cosmic baryonic gas, the evolution of vorticity is also hierarchical. The vorticity evolves from large scales to small scales, and finally falls into massive halos to form structures, including light-emitting objects. This result strongly indicates that the highly nonlinear evolution would lead to the cosmic baryon fluid reaching a statistically quasiequilibrium state satisfying the universal scaling as that of fully developed turbulence.

In view of this picture, we can say that in the highly nonlinear regime, the statistical properties, especially the intermittent behavior, of the velocity fluctuations are actually independent of the details of the dissipative processes. The state depends only on the dimension of dissipative structures and the hierarchical relation index.

\section{Observable Effects}

The nonlinearly evolved IGM provides a coherent and uniform explanation of various observations, including the intermittence and non-Gaussianity of Ly $\alpha$ transmitted fllux of quasar absorption spectrum [44, 45], Ly $\alpha$ leaks of absorption spectrum of high redshift objects [46], the turbulence broadening [47], Scaling relations between SZ effect and X-ray luminosity of X-ray clusters [38, 48, 49], baryon missing in collapsed halos [19], and so forth. Here we address only two of them.

5.1. Non-Gaussianity of Log-Poisson Hierarchy. It has been shown that the SL scaling is yielded from the Log-Poisson hierarchy process, which is related to the so-called generalized scale covariance of the Navier-Stokes equations [50]. Therefore, the statistical behavior of the mass field of cosmic baryon matter should be given by the log-Poisson random multiplicative processes (RMP).

The log-Poisson RMP assumes that, in the scale-free range, the variables $\delta \rho_{r} \equiv \rho(\mathbf{x}+\mathbf{r})-\rho(\mathbf{x}), r=|\mathbf{r}|$, on different scales $r$ are related from each other by a statistically hierarchy relation as

$$
\left|\delta \rho_{r_{2}}\right|=W_{r_{1} r_{2}}\left|\delta \rho_{r_{1}}\right|
$$

where

$$
W_{r_{1} r_{2}}=\beta^{m}\left(\frac{r_{1}}{r_{2}}\right)^{\gamma},
$$

which describes how the fluctuation $\left|\delta \rho_{r_{1}}\right|$ on the larger scale $r_{1}$ is related to fluctuations $\left|\delta \rho_{r_{2}}\right|$ on the smaller scale $r_{2}$. In (26), $m$ is a Poisson random variable with the PDF

$$
P(m)=\exp \left(-\lambda_{r_{1} r_{2}}\right) \frac{\lambda_{r_{1} r_{2}}^{m}}{m !} .
$$

To insure the normalization $\left\langle W_{r_{1} r_{2}}\right\rangle=1$, where $\langle\cdots\rangle$ is over $\mathrm{m}$, the mean $\lambda_{r_{1} r_{2}}$ of the Poisson distribution should be

$$
\lambda_{r_{1} r_{2}}=\frac{\gamma\left[\ln \left(r_{1} / r_{2}\right)\right]}{1-\beta} .
$$

It is enough to consider only $\left|\delta \rho_{r}\right|$, as the distribution of positive and negative $\delta \rho_{r}$ is symmetric.

The log-Poisson hierarchy given by (26) depends only on the ratio $r_{1} / r_{2}$, which is obviously scale invariant. The hierarchy is determined by two dimensionless positive parameters: 
$\beta$ and $\gamma$, describing, respectively, the intermittence and singularity of the random fields. Equation (26) relates $\delta \rho_{r}$ on different scales by multiplying a random factor $W$, which generally yields a non-Gaussian field even if the field originally is Gaussian [51].

The cascade from scale $r_{1}$ to $r_{2}$, and then to $r_{3}$ is identical to the cascade from $r_{1}$ to $r_{3}$. It is because $W_{r_{1} r_{3}}=$ $W_{r_{1} r_{2}} W_{r_{2} r_{3}}=\beta^{N}\left(r_{1} / r_{3}\right)^{\gamma}$, where $N$ is again a Poisson random variable with $\lambda_{r_{1} r_{3}}=\lambda_{r_{1} r_{2}}+\lambda_{r_{2} r_{3}}=\gamma\left[\ln \left(r_{1} / r_{3}\right)\right] /(1-\beta)$. Therefore, the log-Poisson hierarchy removes an arbitrariness in defining the steps of cascade from $r_{1}$ to $r_{2}$ or $r_{2}$ to $r_{3}$. The log-Poisson hierarchy is discrete in terms of the discrete random number $m$. However, the scale $r$ is infinitely divisible. Namely, there is no lower limit on the difference $r_{1}-r_{2}$. It can be infinitesimal, and the hierarchical process is of infinite divisibility.

With the log-Poisson hierarchy (25)-(28), the density field should have the non-Gaussianities, which can be described by the structure function of density field $S_{p}(r) \equiv$ $\left\langle\left|\delta \rho_{r}\right|^{p}\right\rangle$ as follows [52].

(1) Intermittent Exponent. The structure function should be a function of power law of $r$ as

$$
S_{p}(r) \propto r^{\xi(p)},
$$

and the intermittent exponent $\xi(p)$ is given by

$$
\xi(p)=-\gamma\left[p-\frac{\left(1-\beta^{p}\right)}{1-\beta}\right] .
$$

This is actually the SL scaling formula for density field.

\section{(2) Hierarchical Relation. Defining}

$$
F_{p}(r) \equiv \frac{S_{p+1}(r)}{S_{p}(r)},
$$

the log-Poisson RMP gives hierarchical relation as

$$
\frac{F_{p}(r)}{F_{\infty}(r)}=\left[\frac{F_{p+1}(r)}{F_{\infty}(r)}\right]^{1 / \beta} .
$$

Relation (32) is invariant with respect to a translation in $p$.

(3) Moments. The ratio between high-order and 2nd-order moments, $\left\langle\delta \rho_{r}^{2 p}\right\rangle /\left\langle\delta \rho_{r}^{2}\right\rangle^{p}$, should satisfy

$$
\ln \frac{\left\langle\delta \rho_{r}^{2 p}\right\rangle}{\left\langle\delta \rho_{r}^{2}\right\rangle^{p}}=K_{p} \ln r+\text { const }
$$

with

$$
K_{p}=-\gamma \frac{p\left(1-\beta^{2}\right)-\left(1-\beta^{2 p}\right)}{1-\beta} .
$$

(4) Scale-Scale Correlation. The so-called scale-scale correlation is defined as

$$
C_{r_{1}, r_{2}}^{p, p} \equiv \frac{\left\langle\delta \rho_{r_{1}}^{p} \delta \rho_{r_{2}}^{p}\right\rangle}{\left\langle\delta \rho_{r_{1}}^{p}\right\rangle\left\langle\delta \rho_{r_{2}}^{p}\right\rangle} .
$$

Obviously, for a Gaussian field, $C_{r_{1}, r_{2}}^{p, p}=1$. If the ratio $r_{2} / r_{1}$ is fixed, the log-Poisson model predicts the scale-scale correlation to be

$$
C_{r_{1}, r_{2}}^{p, p}=B\left(\frac{r_{2}}{r_{1}}\right) r_{1}^{\xi(2 p)-2 \xi(p)}
$$

where the coefficient $B\left(r_{2} / r_{1}\right)$ depends only on the ratio $r_{2} / r_{1}$, as the log-Poisson model is invariant of the dilation.

It should be emphasized again that all above-mentioned non-Gaussianities depend only on two dimensionless parameters $\beta$ and $\gamma$. It has been revealed that the fields of Ly $\alpha$ transmitted flux fluctuations of observed high resolution and high S/N Ly $\alpha$ absorption spectra of quasars at moderate redshift $z \sim 2-3$ and high redshift $z \sim 5-6$ do show all the logPoisson non-Gaussianities [53, 54].

5.2. Turbulence Pressure and Baryon Missing. As shown in Section 2.2, the Jeans length $\lambda_{J}=2 \pi / k_{J}$ is $\lambda_{J}=c_{s} \sqrt{\pi / G \rho}$, with $c_{s}$ being the sound speed. The effect of turbulent motions on the Jeans length can be estimated by replacing $c_{s}$ by an effective sound speed

$$
c_{s, \mathrm{eff}}^{2}=c_{s}^{2}+\frac{1}{3}\left\langle v^{2}\right\rangle
$$

where $\left\langle v^{2}\right\rangle$ is the rms velocity dispersion due to turbulent motion $[55,56]$. Namely, the random velocity field of turbulence contributes to an extra pressure, the turbulence pressure, $p_{\mathrm{tub}}=\rho\left\langle v^{2}\right\rangle$ to resist the gravitational collapsing.

If the collapsed halo is on scale $R$, the gravitational collapsing will not be affected by the rms velocity dispersion on scales larger than $R$, and the fluctuation of velocity on the scales $k<2 \pi / R$ does not contribute to the turbulent pressure in terms of resist gravitational collapsing on scales that larger than $R$. Thus, the effect of turbulent pressure on gravitational clustering should be given by [57]

$$
p_{\text {tur }}\left(k_{R}\right)=\int_{k_{R}}^{k_{\max }} E(k) d k,
$$

where $E(k)$ is the power spectrum of kinetic energy of the turbulence, and $k_{R}=2 \pi / R$. Wavenumber $k_{\max }=2 \pi / l_{\text {diss }}$ corresponds to the minimal scale $l_{\text {diss }}$ below which the turbulence decays due to energy dissipation or virialization. The turbulent pressure is dynamical, not thermal. It can be as strong as a thermal pressure of temperature $10^{5-6} \mathrm{~K}$, while the temperature of the IGM is still remained in the range of $10^{4-5} \mathrm{~K}$.

Obviously, the bulk velocity will not contribute to the $\left\langle v^{2}\right\rangle$, as it can be given by the motion of falling into a gravitational well. Therefore, we should pick up the component of velocity field that is regardless of gravity. It 
is mainly given by the vorticity of velocity field. In other words, the turbulent vorticity field can prevent the collapsing of baryon matter into the halos of dark matter. It leads to the deviation of the mass density fields of baryon matter from that of dark matter.

With the samples produced by cosmological hydrodynamical simulation, we find that the turbulence pressure will play an important role of preventing the IGM clustering. In the IGM regions with 10 times mean overdensity, the turbulent pressure is equivalent to the thermal pressure of the baryon gas with temperature of about $1 \times 10^{5} \mathrm{~K}$. The turbulent pressure is dynamical and nonthermal. When the turbulence dissipated, the kinetic energy of turbulence becomes the thermal energy. It yields the entropy in halos [38].

The baryon fraction of dark matter halos is found to be a strong function of the halo mass $M_{200}$, the mass within the range of total mass density larger than $200 \bar{\rho}_{t}$. The baryon fraction is significantly decreasing with the mass of dark halos. For halos with mass $M_{200}=3 \times 10^{10} \mathrm{M}_{\odot}$, the baryon fraction is only about 0.1 of the cosmic mean of the concordance $\Lambda \mathrm{CDM}$ universe, and it further decreases to 0.03 for $M_{200}=10^{10} \mathrm{M}_{\odot}$ halos. This result is well consistent with observed baryon content of galaxies and galaxy groups. Therefore, the turbulent velocity field of baryon fluid would be the major factor leading to the absence of baryon matter in halos with mass less than $10^{11.5} \mathrm{M}_{\odot}$. With this evolution picture, no preheating or reheating mechanisms are needed for explaining baryon missing.

\section{Conclusion}

The nonlinear evolution of cosmic baryon fluid is a central problem of cosmology. The concordance $\Lambda \mathrm{CDM}$ model provides an effective paradigm of formation and evolution of cosmic structures. The dynamical behavior of the CDM component has been successfully revealed with analytical study and N-body numerical simulation. On the other hand, the dynamics of baryon fluid is less understood, especially in the nonlinear regime, because of the involvement of complicated physical processes, such as cooling, heating, ionization, shock, and turbulence. On the other hand, many observations are directly related to the nonlinear evolution of baryon fluid, such as, the absorption spectra of quasars, galaxies and GRB at high redshift, Sunyaev-Zeldovich effect, the polarization of cosmic microwave background (CMB), $\mathrm{X}$-ray background and so forth. One of the biggest challenges in galaxy formation is also on the physical origin of the nonlinear relation between baryon gas and low-mass dark halos.

Thanks to the development of reliable cosmological hydrodynamical simulation in the last decade, significant progress has been made in understanding the nonlinear dynamics of cosmic baryon fluid. The turbulence behavior of cosmic baryon fluid in scale-free regime is the key of understanding nonlinear evolution of the mass density and velocity fields. An important consequence of the turbulence is to yield statistical decoupling of the mass and velocity fields of baryon fluid from the underlying dark matter field. This result is extremely valuable to understand the low baryon fraction in galaxy clusters, the non-Gaussian features of quasar Ly $\alpha$ transmitted flux, the Ly $\alpha$ leaking of absorption spectra of high redshift objects, the shock heating of previrialized structures, and the strongly nonlinear dependence of the star formation efficiency on halo mass, and so forth. Considering these complexities, the nonlinear dynamics of the IGM also is essential for understanding the epoch of reionization and related observations.

\section{References}

[1] M. Fukugita, C. J. Hogan, and P. J. E. Peebles, "The cosmic baryon budget," Astrophysical Journal, vol. 503, no. 2, pp. 518530, 1998.

[2] R. Cen and J. P. Ostriker, "Where are the baryons?" Astrophysical Journal, vol. 514, no. 1, pp. 1-6, 1999.

[3] R. Davé, R. Cen, J. P. Ostriker et al., "Baryons in the warm-hot intergalactic medium," Astrophysical Journal, vol. 552, no. 2, pp. 473-483, 2001.

[4] J. Dunkley, E. Komatsu, M. R. Nolta et al., "Five-year wilkinson microwave anisotropy probe observations: likelihoods and parameters from the WMAP data," Astrophysical Journal, Supplement Series, vol. 180, no. 2, pp. 306-329, 2009.

[5] E. Komatsu, J. Dunkley, M. R. Nolta et al., "Five-year wilkinson microwave anisotropy probe observations: cosmological interpretation," Astrophysical Journal, Supplement Series, vol. 180, no. 2, pp. 330-376, 2009.

[6] S. Ettori, "Are we missing baryons in galaxy clusters?" Monthly Notices of the Royal Astronomical Society, vol. 344, no. 2, pp. L13-L16, 2003.

[7] X. Y. Dai, J. N. Bregman, C. S. Kochanek, and E. Rasia, "On the baryon fractions in clusters and groups of galaxies," Astrophysical Journal, vol. 719, no. 1, article 119, 2010.

[8] S. Giodini, D. Pierini, A. Finoguenov et al., "Stellar and total baryon mass fractions in groups and clusters since redshift," Astrophysical Journal, vol. 703, no. 1, pp. 982-993, 2009.

[9] S. S. McGaugh, J. M. Schombert, W. J. G. de Blok, and M. J. Zagursky, "The baryon content of cosmic structures," Astrophysical Journal, vol. 708, no. 1, part 2, pp. L14-L17, 2010.

[10] A. Dekel and J. Silk, "The origin of dwarf galaxies, cold dark matter, and biased galaxy formation," Astrophysical Journal, vol. 303, pp. 39-55, 1986.

[11] D. J. Croton, V. Springel, S. D. M. White et al., "The many lives of active galactic nuclei: cooling flows, black holes and the luminosities and colours of galaxies," Monthly Notices of the Royal Astronomical Society, vol. 365, no. 1, pp. 11-28, 2006.

[12] R. S. Somerville, P. F. Hopkins, T. J. Cox, B. E. Robertson, and L. Hernquist, "A semi-analytic model for the co-evolution of galaxies, black holes and active galactic nuclei," Monthly Notices of the Royal Astronomical Society, vol. 391, no. 2, pp. 481-506, 2008.

[13] A. C. da Silva, S. T. Kay, A. R. Liddle, and P. A. Thomas, "Hydrodynamical simulations of the Sunyaev-Zel'dovich effect: cluster scaling relations and X-ray properties," Monthly Notices of the Royal Astronomical Society, vol. 348, no. 4, pp. 1401-1408, 2004.

[14] H. J. Mo, X. Yang, F. C. van den Bosch, and N. Katz, "Pre-heating by pre-virialization and its impact on galaxy formation," Monthly Notices of the Royal Astronomical Society, vol. 363, no. 4, pp. 1155-1166, 2005. 
[15] M.-M. M. Low and A. Ferrara, "Starburst-driven mass loss from dwarf galaxies: efficiency and metal ejection," Astrophysical Journal, vol. 513, no. 1, pp. 142-155, 1999.

[16] R. A. Crain, V. R. Eke, C. S. Frenk et al., "The baryon fraction of $\Lambda \mathrm{cDM}$ haloes," Monthly Notices of the Royal Astronomical Society, vol. 377, no. 1, pp. 41-49, 2007.

[17] D. Kereš, N. Katz, R. Davé, M. Fardal, and D. H. Weinberg, "Galaxies in a simulated $\Lambda \mathrm{cDM}$ universe-II. Observable properties and constraints on feedback," Monthly Notices of the Royal Astronomical Society, vol. 396, no. 4, pp. 2332-2344, 2009.

[18] S. F. Shandarin and Ya. B. Zeldovich, "The large-scale structure of the universe: turbulence, intermittency, structures in a selfgravitating medium," Reviews of Modern Physics, vol. 61, no. 2, pp. 185-220, 1989.

[19] P. He, L.-L. Feng, and L.-Z. Fang, "Distributions of the baryon fraction on large scales in the universe," Astrophysical Journal, vol. 623, no. 2, pp. 601-611, 2005.

[20] I. Wasserman, "On the origin of galaxies, galactic angular momenta, and galactic magnetic fields," Astrophysical Journal, vol. 224, pp. 337-343, 1978.

[21] H. G. Bi, G. Börne, and Y. Q. Chu, "An alternative model for the Ly-alpha absorption forest," Astronomy and Astrophysics, vol. 266, no. 1, pp. 1-5, 1992.

[22] A. Nusser, "Analytic solutions for coupled linear perturbations," Monthly Notices of the Royal Astronomical Society, vol. 317, no. 4, pp. 902-906, 2000.

[23] S. Matarrese and R. Mohayaee, "The growth of structure in the intergalactic medium," Monthly Notices of the Royal Astronomical Society, vol. 329, no. 1, pp. 37-60, 2002.

[24] O. Lahav, P. B. Lilje, J. R. Primack, et al., "Dynamical effects of the cosmologcal constant," Monthly Notices of the Royal Astronomical Society, vol. 251, pp. 128-136, 1991.

[25] M. Kardar, G. Parisi, and Y.-C. Zhang, "Dynamic scaling of growing interfaces," Physical Review Letters, vol. 56, no. 9, pp. 889-892, 1986.

[26] S. N. Gurbatov, A. I. Saichev, and S. F. Shandarin, "The largescale structures of the universe in the frame of the model equation of non-linear diffusion," Monthly Notices of the Royal Astronomical Society, vol. 236, pp. 385-402, 1989.

[27] A. Berera and L.-Z. Fang, "Stochastic fluctuations and structure formation in the Universe," Physical Review Letters, vol. 72, no. 4, pp. 458-461, 1994.

[28] B. J. T. Jones, "The origin of scaling in the galaxy distribution," Monthly Notices of the Royal Astronomical Society, vol. 307, no. 2, pp. 376-386, 1999.

[29] A. Domíguez, "Study of corrections to the dust model via perturbation theory," Monthly Notices of the Royal Astronomical Society, vol. 334, no. 2, pp. 435-443, 2002.

[30] A. M. Polyakov, "Turbulence without pressure," Physical Review E, vol. 52, no. 6, pp. 6183-6188, 1995.

[31] J. P. Bouchaud, M. Mézard, and G. Parisi, "Scaling and intermittency in Burgers turbulence," Physical Review E, vol. 52, no. 4, pp. 3656-3674, 1995.

[32] V. Yakhot, "Probability density and scaling exponents of the moments of longitudinal velocity difference in strong turbulence," Physical Review E, vol. 57, no. 2, pp. 1737-1751, 1998.

[33] M. Lässig, "Dynamical anomalies and intermittency in burgers turbulence," Physical Review Letters, vol. 84, no. 12, pp. 26182621, 2000.

[34] J. Davoudi, A. A. Masoudi, M. R. R. Tabar, A. R. Rastegar, and F. Shahbazi, "Three-dimensional forced Burgers turbulence supplemented with a continuity equation," Physical Review E, vol. 63, no. 5, Article ID 056308, 13 pages, 2001.

[35] L.-L. Feng, J. Pando, and L.-Z. Fang, "Intermittent features of the quasar Ly $\alpha$ transmitted flux: results from cosmological hydrodynamic simulations," Astrophysical Journal, vol. 587, no. 2, pp. 487-499, 2003.

[36] B. Kim, P. He, J. Pando, L.-L. Feng, and L.-Z. Fang, "The velocity field of baryonic gas in the Universe," Astrophysical Journal, vol. 625, no. 2, pp. 599-612, 2005.

[37] W. Zhu, L.-L. Feng, and L.-Z. Fang, "Vorticity of intergalactic medium velocity field on large scales," Astrophysical Journal, vol. 712 , no. 1 , pp. 1-13, 2010.

[38] P. He, L.-L. Feng, and L.-Z. Fang, "Temperature and entropy fields of baryonic gas in the Universe," Astrophysical Journal, vol. 612, no. 1, pp. 14-27, 2004.

[39] G. K. Batchelor, Theory of Homogeneous Turbulence, Cambridge University Press, Cambridge, UK, 1959.

[40] W. Schmidt, "The numerical simulation of turbulence," http://arxiv.org/abs/0712.0954.

[41] A. Kolmogorov, "The local structure of turbulence in incompressible viscous fluid for very large Reynolds' numbers," Doklady Akademii Nauk SSSR, vol. 30, pp. 301-305, 1941.

[42] Z.-S. She and E. Leveque, "Universal scaling laws in fully developed turbulence," Physical Review Letters, vol. 72, no. 3, pp. 336-339, 1994.

[43] P. He, J. Liu, L.-L. Feng, C.-W. Shu, and L.-Z. Fang, "Lowredshift cosmic baryon fluid on large scales and she-leveque universal scaling," Physical Review Letters, vol. 96, no. 5, Article ID 051302, 4 pages, 2006.

[44] P. Jamkhedkar, H. Zhan, and L.-Z. Fang, "The intermittent behavior of the cosmic mass field revealed by a QSO's Ly $\alpha$ forest," Astrophysical Journal, vol. 543, no. 1, pp. L1-L4, 2000.

[45] P. Jamkhedkar, L.-L. Feng, W. Zheng, D. Kirkman, D. Tytler, and L.-Z. Fang, "Power spectrum and intermittency of the transmitted flux from the Lyman $\alpha$ absorption spectra of quasi-stellar objects," Monthly Notices of the Royal Astronomical Society, vol. 343, no. 4, pp. 1110-1122, 2003.

[46] J. Liu, H. Bi, and L.-Z. Fang, "Ly $\alpha$ leaks in the absorption spectra of high-redshift quasi-stellar objects," Astrophysical Journal, vol. 671, no. 2, pp. L89-L92, 2007.

[47] J. Liu, P. Jamkhedkar, W. Zheng, L.-L. Feng, and L.-Z. Fang, "A unified fitting of $\mathrm{H}$ I and He II Ly $\alpha$ transmitted flux of QSO HE 2347-4342 with $\Lambda$ CDM hydrodynamic simulations," Astrophysical Journal, vol. 645, no. 2, pp. 861-867, 2006.

[48] T.-J. Zhang, J. Liu, L.-L. Feng, Ping He, and L.-Z. Fang, "Xray emission of baryonic gas in the universe: luminositytemperature relationship and soft-band background," Astrophysical Journal, vol. 642, no. 2, pp. 625-635, 2006.

[49] Q. Yuan, H.-Y. Wan, T.-J. Zhang, J.-R. Liu, L.-L. Feng, and L.Z. Fang, "Scaling relation between Sunyaev-Zel'dovich effect and X-ray luminosity and scale-free evolution of cosmic baryon field," New Astronomy, vol. 14, no. 2, pp. 152-159, 2009.

[50] B. Dubrulle, "Intermittency in fully developed turbulence: logpoisson statistics and generalized scale covariance," Physical Review Letters, vol. 73, no. 7, pp. 959-962, 1994.

[51] J. Pando and L.-Z. Fang, "Detecting the non-Gaussian spectrum of QSO's Ly $\alpha$ absorption line distribution," Astronomy and Astrophysics, vol. 340, no. 2, pp. 335-342, 1998.

[52] J. R. Liu and L.-Z. Fang, "Non-Gaussianity of the cosmic baryon fluid: log-poisson hierarchy model," Astrophysical Journal, vol. 672, no. 1, pp. 11-18, 2008.

[53] Y. Lu, Y. Q. Chu, and L. Z. Fang, "Log-poission hierarchial clustering of cosmic neutral hydrogen and Ly $\alpha$ transmitted 
flux of QSO absorption spectrum," Astrophysical Journal, vol. 691, no. 1, article 43, 2009.

[54] Y. Lu, W. S. Zhu, Y. Q. Chu, L. L. Feng, and L. Z. Fang, "Logpoisson non-Gaussianity of Ly $\alpha$ transmitted flux fluctuations at high redshift," to appear in Monthly Notices of the Royal Astronomical Society.

[55] S. Chandrasekhar, "The fluctuations of density in isotropic turbulence," Proceedings of the Royal Society of London A, vol. 210, no. 1100, pp. 18-25, 1951.

[56] S. Chandrasekhar, "The gravitational instability of an infinite homogeneous turbulent medium," Proceedings of the Royal Society of London A, vol. 210, no. 1100, pp. 26-29, 1951.

[57] S. Bonazzola, J. Heyvaerts, E. Falgarone, et al., "Jeans collapse in a turbulent medium," Astronomy and Astrophysics, vol. 172, no. 1-2, pp. 293-298, 1987. 

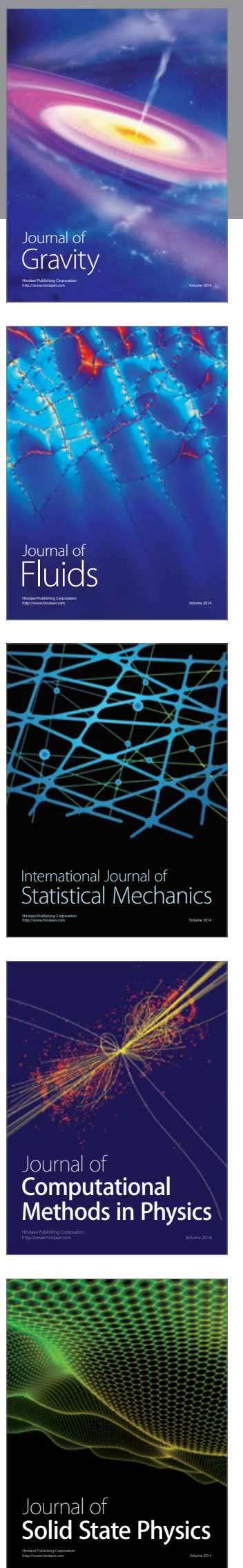

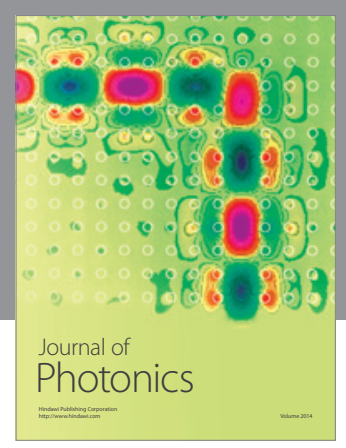

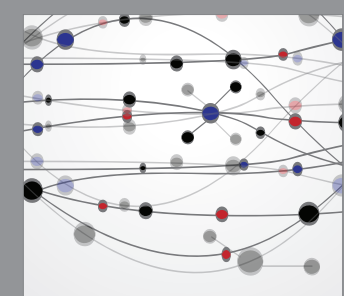

The Scientific World Journal
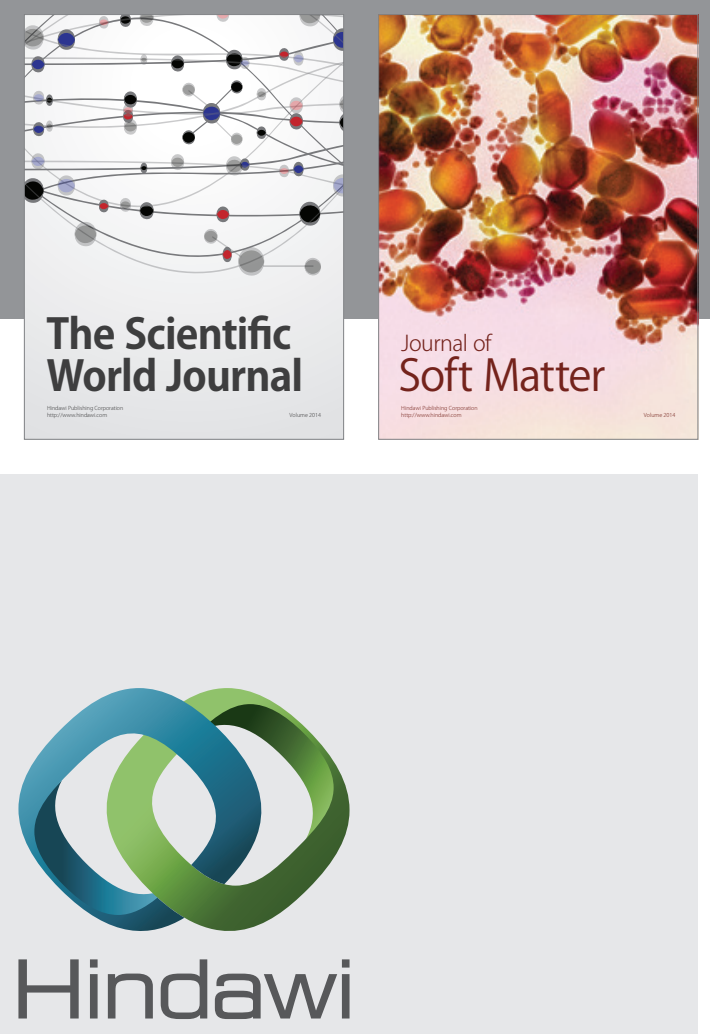

Submit your manuscripts at

http://www.hindawi.com
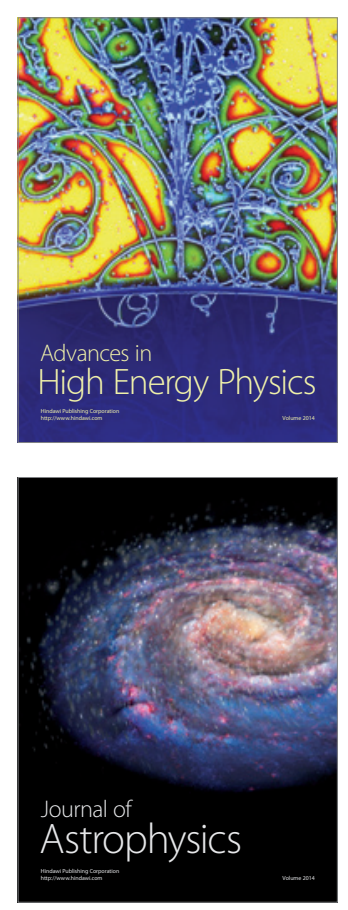
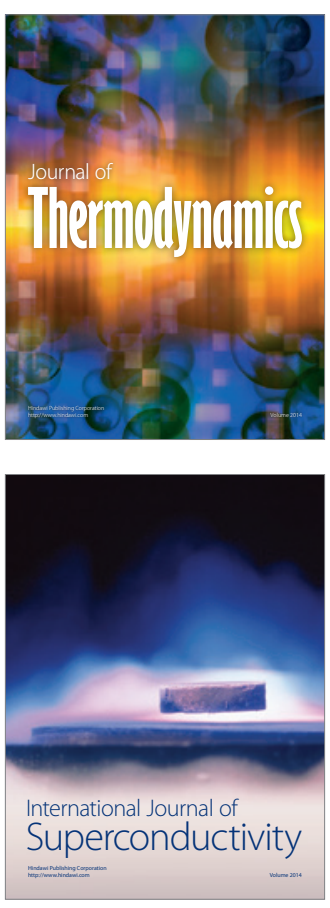
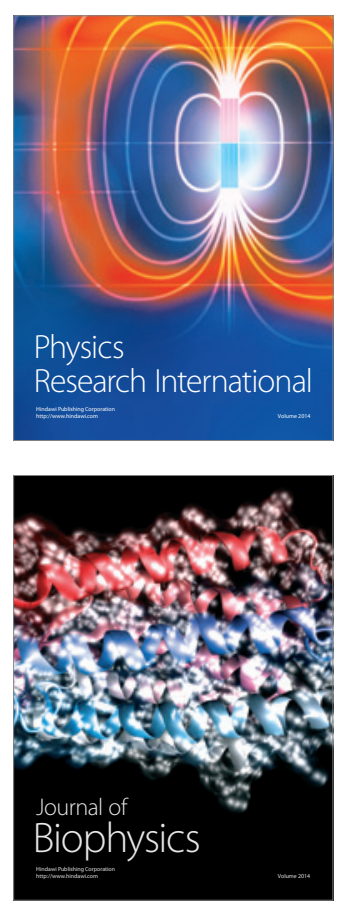
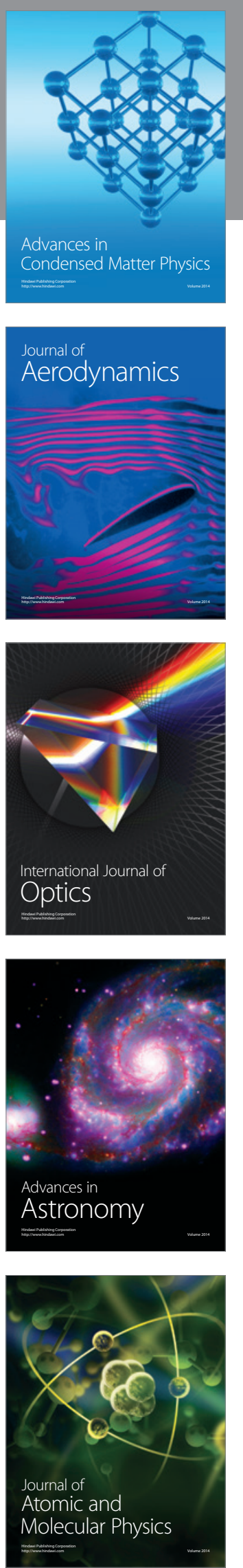